\title{
Anti-diabetic treatment as an additional factor in a FRAX based evaluation of osteoporotic fracture risk
}

Maria P. Yavropoulou, Athanasios Mousiolis, Basiliki Kolokouri, Pelagia Kolibianaki, Athina Dimitriou, Petros Papaleksis, Michalis Daniilidis, Kaliopi Kotsa

Division of Endocrinology, 1st Department of Internal Medicine, AHEPA hospital, Thessaloniki, Greece

\section{Background:}

The present study is designed to assess the incidence of osteoporotic fractures and the associated risk factors and particularly those used to predict the 10-year fracture risk in FRAX score based on data gathered in general practitioner's records of rural Greece.

\section{Patients and methods}

We conducted a retrospective analysis of all patients with osteoporosis presented between October 2013 and December 2014. Data from medical records including gender, age, previous history of low energy fractures (spine, and distal radius), past medical history, and medication use with specific reference to treatment with bisphosphonates and glucocorticoids were obtained. Patients with metabolic bone disease other than osteoporosis were excluded from the final analysis.

\section{Results}

One hundred and sixty seven patients (127 women and 40 men) aged between 44 and 90 years old were included in the final analysis. Twenty seven per cent of the study population ( $n=45$ ) had sustained a low energy fracture (Figure 1.) and only $43 \%$ of them had received anti-osteoporosis treatment (Figure 2.). Regarding concomitant medications only anti-diabetic treatment was significantly associated with the presence of osteoporotic fracture $(F=4,260, p=0.042)$, and had a considerable effect on the 10-year risk of major osteoporotic and hip fractures in drug-naïve patients.

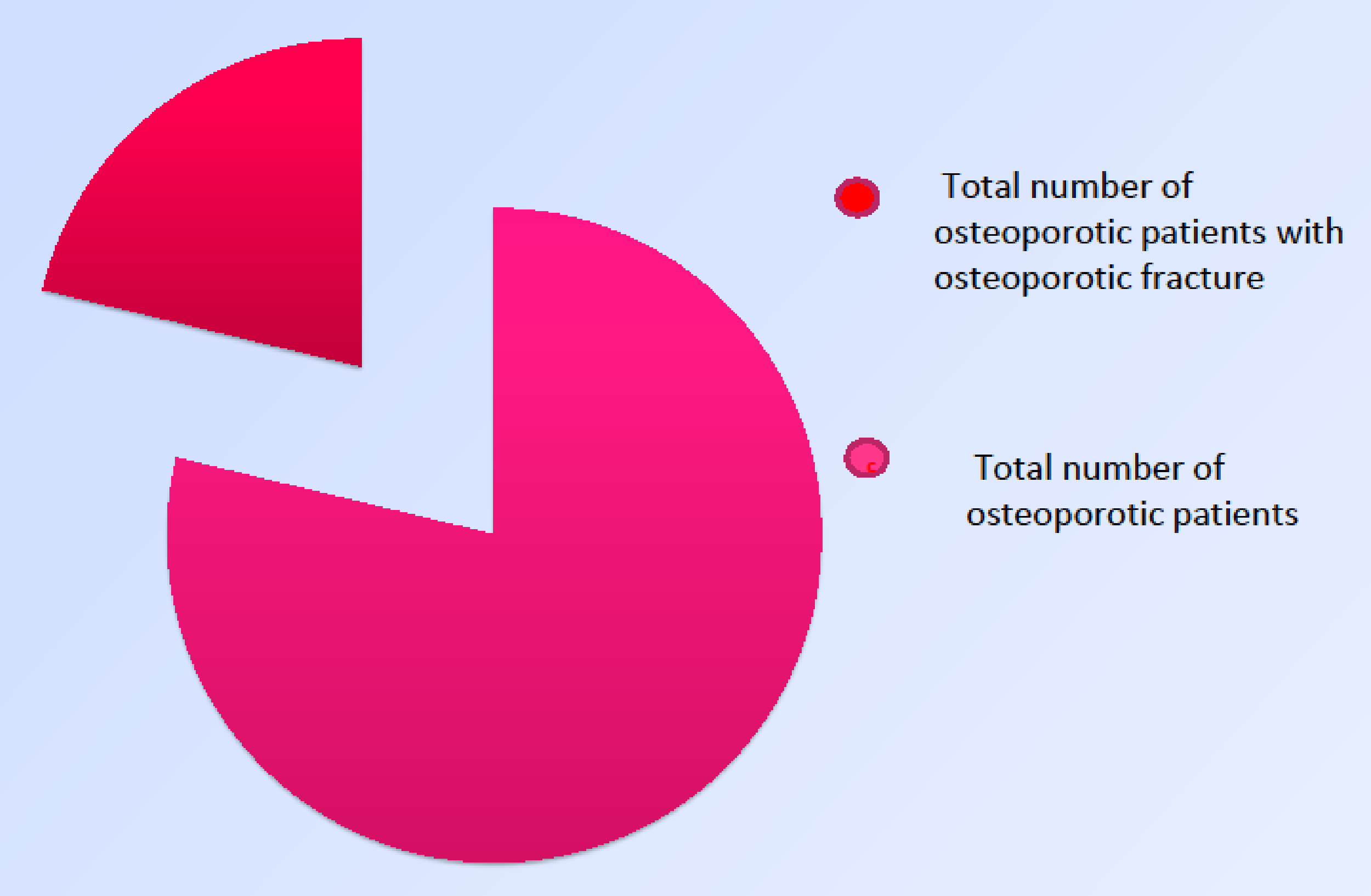

Figure 1. Percentage of patients with osteoporotic fracture

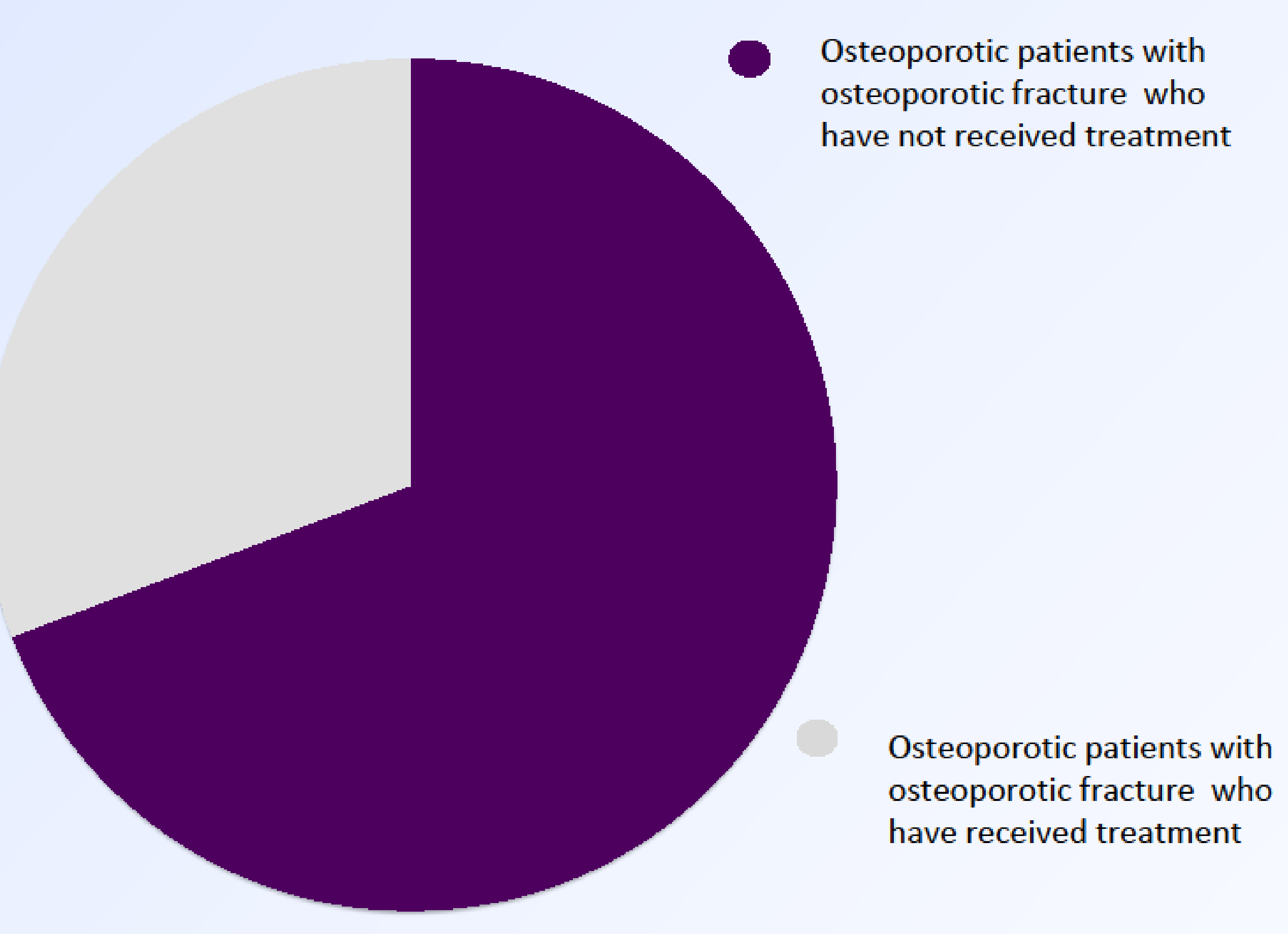

Figure 2. Percentage of patients with osteoporotic Fracture that have received treatment

\section{Conclusion}

Anti-diabetic treatment should be taken into consideration when evaluating fracture risk in osteoporotic patients. 